\title{
Zur Bewertung der Ärzteschaft und des medizinischen Wissens in einem Gesund heitsforum
}

\section{How Physicians and Medical Knowledge is Assessed in a Health Forum}

\author{
Hans GIESSEN ${ }^{1}$
}

Prof. Dr., Universität des Saarlandes, Zentrum für lebenslanges Lernen, Saarbrücken, Germany

ORCID: H.G. 0000-0002-4024-1664

\section{Corresponding author:}

Hans GIESSEN,

Universität des Saarlandes, Zentrum für lebenslanges Lernen, Campus,

Saarbrücken, Germany

E-mail: h.giessen@is.uni-sb.de

Submitted: 05.01 .2020

Revision Requested: 06.01.2020

Last Revision Received: 26.03.2020

Accepted: 26.03 .2020

Citation: Giessen, H. (2020). Zur Bewertung der Ärzteschaft und des medizinischen Wissens in einem Gesundheitsforum. Alman Dili ve Edebiyatı Dergisi - Studien zur deutschen Sprache und Literatur, 43, 1-17.

https://doi.org/10.26650/sdsl2020-0002

\begin{abstract}
DEUTSCH)
Das Web 2.0 wird oft als "sozial" oder partizipativ bezeichnet. Ein Genre des Web 2.0, für das dies sicherlich und vom Grundsatz her uneingeschränkt gilt, sind Foren. Jeder Benutzer kann am Kommunikationsaustausch teilnehmen und sein Wissen, seine Erfahrungen und seine Meinung frei und unbegrenzt veröffentlichen. Diese Studie konzentriert sich daher auf das Genre der Foren. Ich habe die Besonderheiten einer solchen Laienkommunikation im Kontext eines bestimmten Forums qualitativ und nach diskurs- oder inhaltsanalytischen Kriterien analysiert. Inhaltlich beziehe ich mich auf ein Forum aus Deutschland, das sich mit Gesundheitsfragen befasst. Ziel war es zu untersuchen, wie medizinisches Fachpersonal und medizinisches Wissen geschätzt werden. Da es im Kontext eines solchen Forums schwierig ist, auf eine genaue Anzahl von Benutzern zuzugreifen, habe ich eine bestimmte Website ausgewählt, die in der Liste der am häufigsten besuchten Websites aufgeführt ist und die aufgrund ihrer URL bereits eine besondere Wahrscheinlichkeit für den Zugriff aufweist (daher: der Name): www.gesundheit.de (laut rechtlichem Hinweis wird dieses Forum von Alliance Healthcare Germany mit Sitz in Frankfurt am Main betrieben). Die Forenbeiträge wurden dann aufgenommen und untersucht, wenn der jeweils letzte Forenbeitrag in der Zeit zwischen Dezember 2018 und April 2019 veröffentlicht wurde. Die Beiträge wurden unverändert übernommen, $d$. h. einschließlich der im Original gefundenen orthografischen und grammatikalischen Fehler.
\end{abstract}

Schlüsselwörter: Laienkommunikation, Forenkommunikation,

Gesundheitskommunikation, Internetkommunikation, Medizin-Kommunikation

\section{ABSTRACT (ENGLISH)}

Web 2.0 is often referred to as 'social' or participatory. A genre of Web 2.0 to which this certainly applies is forums. Every user can take part in communication exchange and publish his or her knowledge, experience and his or her very own opinions. This study focuses on the genre of forums. I qualitatively (and according to discourse- or content-analytical criteria) analyzed the special features of such lay communication in the context of a specific forum. In terms of content, I am referring to a forum from 
Germany that deals with health issues. The aim was to examine how medical professionals and medical knowledge are valued. Since it is difficult to access exact numbers of users in the context of such a forum, I selected a specific website that appears in the list of the most visited websites, and that already has a special probability of being accessed due to its URL (hence the name): www.gesundheit.de (according to the legal notice, this forum is operated by Alliance Healthcare Germany, based in Frankfurt am Main). The topics were selected from posts published between December 2018 and April 2019. The contributions were transferred unchanged, i.e. including the orthographic and grammatical errors found in the original. Keywords: Forum communications, Health communications, Internet communications, Layperson communications, Medical communications

\section{EXTENDED ABSTRACT}

Web 2.0 is often referred to as 'social' or participatory. A genre of Web 2.0 to which this certainly applies is forums. Every user can take part in the communication exchange and publish his or her knowledge, experience and his or her very own opinions. This study focuses on the genre of forums. I qualitatively (and according to discourse or contentanalytical criteria) analyzed the special features of such lay communication in the context of a specific forum.

In terms of content, I am referring to a forum from Germany that deals with health issues. The aim was to examine how medical professionals and medical knowledge are valued. Since it is difficult to access exact numbers of users in the context of such a forum, I selected a specific website that appears in the list of the most visited websites, and that already has a special probability of being accessed due to its URL (hence the name): www.gesundheit.de (according to the legal notice this forum is operated by Alliance Healthcare Germany, based in Frankfurt am Main). The topics were selected from posts published between December 2018 and April 2019. The contributions were transferred unchanged, i.e. including the orthographic and grammatical errors found in the original.

It was reasonable to assume that a forum in which users meet to inform themselves about diseases in order to prepare themselves for or to follow-up visits at the doctor will also be looking for alternative treatments and that users who are disappointed with medical reactions will express themselves accordingly. Thus, it is the medium itself that suggests that critical positions on doctors, but possibly on clinical medicine as a whole, may dominate.

The result, however, was that there is broad basic trust in medicine, at least in Germany. Against the background described, this is quite surprising. In the context of the forum examined, there was also a fundamental acceptance of the medical system as a 
whole. Whilst the purpose of this forum was assumed to be to absorb criticism and to seek alternative opinions (and, if necessary, to seek alternative therapy), there was little to no fundamental criticism. Thus, whilst this forum seems to be designed with the intent of enabling the expression of anger and frustration, there still is a dominant acceptance of medical science and even the medical profession. There is rarely criticism of individual doctors, too. If an individual physician is criticized, the authors in this forum keep their criticism with the respective doctors and very rarely propose alternative healing methods. Even with medication, there is little or no rejection of standard or conventional medicine.

My hypothesis is that the function and associated seriousness that is expressed in the name of the forum may thus lead to an indeed fundamentally medicine-oriented form of discussion.

As a research article, the paper of course does not and doesn't want to make any assumptions about the reasons why (at least in the context of this forum) rationality is so fundamentally accepted. As I said, alternative methods are almost irrelevant, be they are close to the medical system, like homeopathy; be they remote to the system, such as magic. As a research article, the paper is limited to examining the facts and describing the dominance of the scientific belief system even in situations of crises and under unpleasant to lethal conditions.

Another not insignificant observation is that in almost all contributions on this health forum, the assessment of the trustworthiness and the expertise of the forum authors were particularly important. This is obviously not just about the assessment of the communication partners, but also about the picture that one gives about oneself. Contributors are probably afraid of only being taken seriously if they can credibly demonstrate their own dismay or their own expertise. This may lead to problems in the relationship between 'actual experts' (i.e. medical professionals) and laypersons. However, here, too, it was found that surprisingly few problems have arisen. 


\section{Einleitung}

Nachdem Tim Berners-Lee und Robert Cailliau im März 1989 mit der Entwicklung des HTML-Programmiercodes, des http-Datentransfer-Protokolls und der Identifikatoren-URL am CERN in Genf das Word Wide Web kreiert hatten (Gillies \& Cailliau, 2000), wandelte sich der mediale Informationsaustausch gravierend; insbesondere wurden durch die Gestaltung zahlreicher Websites aus den unterschiedlichsten Bereichen vielfältige Informationen weltweit auffindbar. Mit der Möglichkeit, über Rückkanaloptionen auf den Informationstransfer zu reagieren, erweiterten sich die Möglichkeiten des Web bereits nach kurzer Zeit erneut so stark, dass O'Reilly (2005) gar von einem Web 2.0 sprach, das durch einen ,sozialen' beziehungsweise ,partizipatorischen' Charakter geprägt sei; der Begriff Web 2.0 wurde rasch aufgegriffen. Charakteristisch für Web 2.0 -Applikationen sind, O’Reilly (2005) zufolge, beispielsweise Wikipedia, wo jeder zum Weltwissen beitragen kann, Blogs (statt simpler statischer Webseiten) - oder das formal sehr ähnliche Genre der Foren. Mit ihrer Hilfe wird es jedem Internet-Nutzer möglich, am Kommunikationsaustausch zu partizipieren und eigene Kenntnisse, Erfahrungen und Meinungen zu publizieren.

In der Folge haben sich auch mehrere Foren etabliert, die sich mit Themen aus dem Bereich der Gesundheitsinformation befassen und die von zahlreichen Internet-Nutzern zumindest in den entwickelteren Ländern seit vielen Jahren regelmäßig aufgesucht werden. Inzwischen wird von solchen Foren gar als einem wichtigem „[m]ediale[n] [Raum] der Experten-Laien-Kommunikation“ (Busch, 2015) gesprochen. In Deutschland, wo die Studie von Albert Busch durchgeführt worden war, erfolgt die Nutzung beispielsweise sehr kontinuierlich. Die Nutzerzahlen sind konstant hoch und nehmen sogar immer noch zu (siehe Bitkom, 2011; Kristiansen \& Bonfadelli, 2013, S. 240-243; Statista, 2017, 2019). Gemäß einer aktuellen Studie der Bertelsmann-Stiftung (2018) informiert sich die Hälfte der deutschen Internetnutzer mindestens einmal im Monat online über Gesundheitsthemen, davon 58 Prozent vor dem Gang zum Arzt. 62 Prozent recherchieren die Diagnose nach dem Praxisbesuch im Internet.

Die Gründe und Motive, sich in Gesundheitsforen einzubringen, sind nach Kaltenborn (2001, S. 50-51) vielschichtig. Relevant seien (1.) die Suche nach Information für krankheitsadäquates Selbstmanagement und für förderliches Gesundheitsverhalten, (2.) die Suche nach Wissen und Information zur Vorbereitung auf den Arztbesuch, (3.) und damit in engem Zusammenhang stehend die Suche nach Information, um angesichts der Vertrauens- / Misstrauensproblematik gegenüber medizinischen Wissensangeboten und 
insbesondere gegenüber dem behandelnden Arzt gewappnet zu sein (mit dem Motiv, in der Lage zu einer kritischen Überprüfung von Diagnosen und Therapien zu sein und insbesondere mit dem Ziel, Zusatzinformationen zu erhalten, die es erlauben, die vom Arzt empfohlenen Handlungsanweisungen verstehen zu können) sowie (4.) - auf einer ganz anderen Ebene - die Suche nach Austausch, denn neben der reinen Informationssuche sei der social support ein ganz wesentlicher Grund für die Nutzung von Gesundheitsforen: die Suche nach Trost, die Diskussion alltäglicher Herausforderungen im Kontext einer Erkrankung, sowie der Erfahrungsaustausch.

Vor dem Hintergrund einer immer intensiveren Nutzung von Gesundheitsforen wird die Frage relevant, welche Konsequenzen dies für die Gesundheitskommunikation und insbesondere für das Arzt-Patienten-Verhältnis und die schwierige Beziehung zwischen medizinischen Experten und Laien hat. Grundsätzlich wird - nicht nur in Bezug auf die deutschsprachigen Länder und nicht nur hinsichtlich der Gesundheitskommunikation davon ausgegangen, dass die sozialen Medien und das Web 2.0 die Beziehung zwischen Experten, Laien und Organisationen verändert habe (Myers, 2015, S. 54) ${ }^{1}$. Generell wird immer wieder diskutiert, ob die neuen Möglichkeiten, die nun auch Laien haben, um an Fach- und Expertendiskussionen teilzunehmen, ein Vorteil oder ein Nachteil darstellen. Dies gilt in besonderem Maße für die Gesundheitskommunikation (z.B. Bromme, Jucks \& Runde, 2005). Auch die Reaktion seitens der Ärzte ist daher ambivalent (BertelsmannStiftung, 2018, S. 8). Im Bericht der Bertelsmann-Stiftung wird zudem darauf verwiesen, dass vielen Nutzern nicht klar sei, ob die Informationen von einer kompetenten Quelle stammten. Es gibt Foren, die von wissenschaftlichen Instituten, Selbsthilfegruppen, Krankenkassen oder Ärzteorganisationen betrieben werden, aber häufig tauschen sich die Teilnehmer ohne beziehungsweise (zumindest) ohne zeitnahe redaktionelle Kontrolle aus.

Eine Beschäftigung mit dem von Sonja Kleinke (2015) so genannten „Laiendiskurs Gesundheit" ist auch deshalb bedeutsam, weil Foren im Bereich der Gesundheitsinformation eine besondere Brisanz aufweisen, denn hier hat Expertenwissen und seine Befolgung oder umgekehrt die Ignoranz von Expertenwissen gegebenenfalls letale Folgen.

Im Folgenden soll daher versucht werden, forengestützte Online-Kommunikation zu sichten und sie bezüglich ihrer Positionierung im Hinblick auf ihre Bewertung der

1 "The many ways social media and the Web 2.0 have transformed relations between professionals, lay people, and organisations" (Myers, 2015, S. 54). 
Ärzteschaft und des medizinischen Wissens zu kategorisieren. Konkret werden Beiträge aus einem besonders verbreiteten deutschsprachigen Online-Forum der Themengebiete Medizin und Gesundheit untersucht, und zu erkennen, mit welchen sprachlichen Strategien sich die Teilnehmer präsentieren.

Die Untersuchung erfolgte qualitativ und zunächst hermeneutisch, also durch tendenziell intuitive Interpretationen, um grundlegende Tendenzen zu erkennen, die dann schematisch geordnet worden sind. Sie folgt damit der theoretischen Begründung hermeneutischen Vorgehens insbesondere durch Dilthey (1900 / 1964) und Gadamer (1960). Die so erschlossenen grundlegenden Tendenzen bilden die Struktur des folgenden Artikels. Die einzelnen Textbeispiele werden in diesem Ordnungsrahmen dann diskurs- beziehungsweise inhaltsanalytisch (vergleiche beispielsweise Angermüller et a. 2001; Kuckarzt, 2016) analysiert.

Die schematische Ordnung bezog sich auf die Bewertung der Ärzteschaft beziehungsweise allgemein der Schulmedizin. Es bietet sich mithin ein relativ klares Raster an, das entsprechend eines Kontinuums darstellt, welche Einstellungen bezüglich der Ärzteschaft beziehungsweise der Schulmedizin vorherrschend sind. Das Kontinuum reicht von der uneingeschränkt positiven Bewertung über unterschiedliche Abstufungen der Kritik (zunächst: an konkreten Ärzten, nicht aber grundsätzlich an der Ärzteschaft beziehungsweise Schulmedizin, über eine generell vorherrschende Skepsis bis hin zur Ablehnung schulmedizinischen Vorgehens und zur Hinwendung zu alternativen Heilmethoden).

Nach der Sichtung und hermeneutischen Interpretation wurden die Forentexte entsprechend dieses Rasters eingeordnet und dann weiter interpretiert.

Das Raster verdeutlicht gleichzeitig auch die Fragestellung, die zur vorliegenden Untersuchung geführt hat. Grundsätzlich geht es darum, mittels des partizipatorischen Web 2.0. die Positionen der Nutzer zu erkennen und herauszuarbeiten. Es kann davon ausgegangen werden, dass es sich jeweils um Personen handelt, die ein akutes medizinisches Problem haben, also persönlich sehr involviert sind. Wie wirkt sich diese persönliche Betroffenheit auf die Einstellungen zur Medizin aus? Konkret: Wie ist die Position dieser direkt Betroffenen zur Schulmedizin? Vertrauen sie ihr und dem medizinischen Personal, gerade angesichts der persönlichen Betroffenheit, oder führt diese Betroffenheit dazu, dass sie sich alternativen medizinischen Angeboten (Esoterik) 
zuwenden? Die persönliche Betroffenheit kann zu besonders authentischen Ergebnissen führen. Damit führen die Beobachtungen mit hoher Validität zu Informationen über die Akzeptanz der Schulmedizin und ihrer Vertreter.

Ziel ist also eine semiotische Studie, die Forenbeiträge als Indikatoren für Einstellungen zur Schulmedizin nutzen möchte.

Fraglich ist die Repräsentativität des möglichen Ergebnisses. Grundsätzlich ist das Vorgehen, mit Hilfe sozialer Äußerungen einer möglichst repräsentativen oder (alternativ) involvierten Personengruppe auf Einstellungen im Kontext dieser Gruppe, aber auch darüber Hinaus auf die öffentliche Meinung (Lippmann, 1922) oder zumindest auf soziale Deutungsmuster der relevanten Teilpublika (Kassner, 2003) zu schließen, nicht neu und wird häufig angewandt. Wichtig ist allerdings, darauf zu achten, dass die Aussagen aus einem Kontext stammen, der entsprechend aussagekräftig ist. Um die Validität der Aussagen zu gewährleisten, wurde hier ein Forum ausgewählt, das in der Liste der meistaufgerufenen Websites erscheint (Alexa, 2019) und das bereits aufgrund seiner URL (mithin der Benennung) eine besondere Wahrscheinlichkeit aufweist, bezüglich der hier interessierenden Thematik angesteuert zu werden: www.gesundheit.de (laut Impressum betrieben von der Alliance Healthcare Deutschland mit Sitz in Frankfurt). Es werden nur solche Forenbeiträge genutzt, die Erkenntnisse über die Identitätskonstruktion und die Selbst- wie die Fremdeinordnung als,Experte' und ,Laie' geben.

Gesichtet wurden Themen, deren letzter Beitrag zwischen Dezember 2018 und April 2019 erschienen ist. Das Korpus besteht also aus der Komplettsammlung dieser Beiträge, die zunächst elektronisch abgespeichert wurden. Danach wurden sie, wie erwähnt, vorgesichtet und hermeneutisch interpretiert sowie in der Folge gemäß des sich am beschriebenen Kontinuum orientierenden Rasters einsortiert. Der folgende Artikel präsentiert nun die in Bezug auf die Fragestellung aussagekräftigsten Beiträge.

Ehrhardt (2011; 2014) und Rentel (2015) charakteristisieren die Kommunikationssituation in Foren als asynchron virtuell, indirekt und dialogisch obwohl das Kriterium der Dialogizität nicht immer zutrifft, denn erstaunlich oft erfolgt auf einen Foreneintrag keine Reaktion, obwohl dies von den Autoren erwartet wird. Zudem variiert die Zahl der Beiträge in Abhängigkeit zum Thema teilweise beträchtlich. Auch die Zeitrahmen sind überraschend groß: Teilweise erfolgt die Reaktion auch erst Wochen nach der ursprünglichen Einstellung im Forum. 
Das für computergestützte Medien typische Auftreten grammatischer und orthographischer Fehler überrascht angesichts der akademischen Thematik, hängt aber wohl von der Medien- und Nutzersituation bei der Erstellung von Texten im Rahmen einer solchen Forenkommunikation ab. Die Forenbeiträge wurden in den im weiteren zitierten Beispielen unverändert übernommen, d.h. auch inklusive der im Original auffindbaren orthographischen und grammatikalischen Fehler.

\section{Qualitative Darstellung der Kommunikationsstrategien}

\subsection{Vorbemerkung}

Gerade aufgrund der teilweise gar existenziellen Themen scheint in fast allen Beiträgen der untersuchten Gesundheitsforen die Einschätzung der Vertrauenswürdigkeit und der Expertise der Forenautoren besonders bedeutsam zu sein; es geht aber offenbar nicht nur um die Einschätzung der Kompetenz und Vertrauenswürdigkeit der antwortenden und Ratschläge erteilenden Kommunikationspartner, sondern auch um das Bild, das man selbst als Suchender beziehungsweise Fragender abgibt. Die Ausgangeshypothese ist, dass man in der jeweiligen Diskussion vermutlich nur ernst genommen wird, wenn man eigene Betroffenheit oder auch eigenes Expertentum glaubwürdig darlegen kann; dieses soziale Element war Anlass der Studie, da daraus die Probleme im Verhältnis, tatsächlicher Experte' (i.e. Mediziner) und Laie erwachsen können. Daraus resultiert die Frage danach, mit welchen sprachlichen Strategien die Forenteilnehmer den Diskurs zu beeinflussen versuchen.

\subsection{Akzeptanz medizinischen Wissens und ärztlichen Expertentums}

\subsubsection{Einführung}

Die Ausgangsvermutung war, dass die Teilnehmer in Gesundheitsforen versuchen, sich Autorität zuzuschreiben, um (als Antwortende auf Fragen) helfen zu können (und um Kritik an eigenen Ratschlägen zu reduzieren); dies gilt aber wohl auch für Fragende, die vermutlich eher relevante Informationen erhalten, wenn sie belegen, dass ihre Anfrage ernsthaft ist und dass sie sich bereits mit der Thematik befasst haben. Fraglich ist, ob das Herausstellen der eigenen Kompetenz auf Kosten der Medizin beziehungsweise der Mediziner erfolgt, oder ob medizinische Kompetenz mit einem Verweis auf die Wissenschaft dargestellt wird. 
Die Sichtung des Korpus hat beides ergeben, wobei die Akzeptanz der Medizin und der Mediziner in diesem Forum deutlich überwiegt. Wenn es zu Kritik an einzelnen Ärzten und ihren Diagnosen kommt, dann häufig deshalb, weil sie aus Sicht der Autoren nicht angemessen medizinisches Wissen weitergegeben oder angewandt haben; dies bedeutet, dass die Relevanz medizinischen beziehungsweise ärztlichen Wissens grundsätzlich akzeptiert wird.

\subsubsection{Referenzieren auf ärztliches Expertentum}

In der Tat versuchen viele Nutzer, ihre Fach- und Urteilskompetenz darzulegen, indem sie auf ärztliches Expertentum verweisen. Quantitativ handelt es sich um die häufigste Strategie - die Nutzer scheinen also mehrheitlich die ärztliche Qualifikation (grundsätzlich) nicht zu hinterfragen.

Die Verweise beziehen sich allgemein auf klinische Studien (Beispiel 1), aber häufig auch auf Mediziner (Beispiel 2), deren Berufsstand man mithin Vertrauen entgegenbringt.

1. aber betrifft sowieso, wie ich gelesen habe, fast 30\% der Bevölkerung (https://www. gesundheit.de/forum/krankheiten-foren/allergie/was-hilft-gegen-allergien-erfahrungen, 13.1.2019)

Die Antwort eines/r Nutzers/in an den Fragesteller verweist auf Mitleidende und hat insofern Trost- und Entlastungsfunktion; abgesichert wird sie mit dem Hinweis darauf, dass entsprechende statistischen Studien der antwortenden Person bekannt sind. Dies wird durch den Nebensatz „wie ich gelesen habe" unterstrichen.

Der Verweis allgemein auf klinische Studien und das medizinische Wissen belegt die hohe Autorität, die die Medizin in diesem Forum geniest. Die bezieht sich natürlich auch auf Mediziner.

2. Wenn es keine Schwellung oder Eiterstellen gibt, die sichtbar sind, hast du sicher eine Art von Problem nicht aber wenn es dir echt so schwer fällt und es Schmerzen gibt - kannst du denn schlucken? - würde ich auf jeden Fall zum Arzt gehen, damit ist nicht zu spaßen. (https://www.gesundheit.de/forum/krankheiten-foren/hals-nasen-ohren/mandelnschmerzen, 3.2.2019) 
Ganz eindeutig ist bei diesem Beispiel „der Arzt" die entscheidende Hilfsinstanz und fachliche Referenz.

\subsubsection{Demonstration eigenen Expertentums bei grundsätzlicher Akzeptanz medizinischen Wissens}

Auch ein Bezug auf eigene Erfahrungen muss keine Kritik an medizinischem Wissen zur Folge haben. Der Rekurs auf eigene Erfahrungen, sei es als mitbetroffene Person, sei es aufgrund einer eigenen Erkrankung, verleiht eine spezifische Legitimation, Ratschläge zu erteilen. Zugleich werden die Glaubwürdigkeit und damit das Vertrauen in den Beitrag erhöht. Der Grad an Subjektivität als Nicht-Fachmann wird durch das Herausstellen langjähriger Vertrautheit mit bestimmten Krankheitsbildern reduziert; individuelle Eindrücke erfahren eine „Verwissenschaftlichung" bzw. Objektivierung, da davon auszugehen ist, dass sich die Betroffenen fundiert über die Erkrankung informiert haben.

3. Hallo Pippes, ich habe auch jahrelang mit Herzrasen in Ruhestellung zu tun gehabt. Bei mir war es ein Mangel an Magnesium. Seit ich jeden Tag Magnesium nehme, habe ich nichts mehr. (https://www.gesundheit.de/forum/krankheiten-foren/herz-kreislauf/ herzrasen-der-nacht, 31.1.2019)

Häufig demonstrieren Autoren des Forums ihre Autorität, indem sie selbst und ohne Hinweis auf Autoritäten jenseits ihrer selbst erläutern, welche ärztlichen Maßnahmen angesichts eines Krankheitsbilds sinnvoll oder gar notwendig sind. In Beispiel 4 wird zunächst nur zu einer Diagnosemethode geraten:

4. du könntest mal auf Darmpilz testen lassen. Oder eine Darmsanierung machen mit anschließendem Wiederaufbau mit Milchsäurebakterien. (https://www.gesundheit.de/ forum/krankheiten-foren/magen-darm/was-stimmt-nur-mit-meinem-darm-nicht, 28.3.2019)

Anschließend wird aber auch eine Therapie sowie eine Rehabilitationsmaßnahme vorgeschlagen. Das sprachliche Vorgehen macht deutlich, dass sich der Autor in der Tat als Experte empfindet und seiner Meinung nach offenbar zu Recht eine selbstverständliche Autorität zum Ausdruck bringt; er sieht sich aufgrund seines Wissensvorsprungs, den er weitergibt, in der Lage, kompetente Ratschläge zu erteilen. Zunächst scheint er sogar zu versuchen, sein Expertentum mittels eines Konjunktivs im ersten Satz (noch) unverbindlichen Ratschlag erscheinen zu lassen. Fraglich ist, ob dies dem Wunsch 
entspricht, als nicht allzu dominant erscheinen zu wollen, oder ob der Konjunktiv analog zum disjunktiven Konjunktion „oder", mit dem der zweite Satz beginnt, lediglich die Alternativen zum Ausdruck bringen soll. Vor diesem Hintergrund ist auch fraglich, wie der Punkt und der zweite Satz, der mit der disjunktiven Konjunktion beginnt, einzuordnen ist. Beim Punkt könnte es sich um einen beim Schreiben mit der Tastatur häufig vorkommenden Tippfehler handeln, so dass der zweite Satz als Nebensatz zu interpretieren wäre, wobei dann das konjunktivische Hilfsverb für beide Sätze gälte. „[M] achen" könnte aber auch ein eigenständiges satzkonstituierendes Verb darstellen, so dass der Autor vom Konjunktiv in den Indikativ wechselt, da er nach dem vorsichtigeren Einstieg nun seine Autorität klar demonstrieren möchte. In jedem Fall ist deutlich, dass der Beitrag das (angenommene oder reale) Expertentum des Autors zum Ausdruck bringt. Andererseits ist ebenso deutlich, dass grundsätzlich medizinisches Wissen und „ärztlichen Maßnahmen“ nicht hinterfragt oder kritisiert werden.

\subsubsection{Referenzieren auf klinische Studien bei gleichzeitiger Kritik an individuellen Ärzten beziehungsweise Diagnosen}

Auch dann, wenn Ärzte kritisiert werden, ist nicht immer eine grundsätzliche Kritik an medizinischem Wissen oder der allgemeinen Notwendigkeit und Kompetenz der Ärzteschaft gegeben.

Mitunter handelt es sich um den Ausdruck von Hilflosigkeit. Aber selbst dann wird nicht notwendigerweise die Kompetenz der Ärzteschaft oder medizinischen Wissens in Frage gestellt - selbst dann nicht, wenn ein/e Ratsuchende/r das Gefühl hat, dass die eigene Hilflosigkeit auf Fehlverhalten von Ärzten zurückzuführen ist, wie im Beispiel 5:

5. dort hat man mir aber nicht wirklich dahingehend zugehört und somit mir auch nichts empfehlen können. (https://www.gesundheit.de/forum/krankheiten-foren/halsnasen-ohren/rachentzuendung-heilt-nicht-ab, 3.4.2019)

Noch dramatischer ist die Kritik an ärztlichen Verhalten in Beispiel 6:

6. Ich soll auch zum Neurologen aber denke es liegt an den augen und werde auch nur als Patient mit Psyschichen Problemen abgestempelt. (https://www.gesundheit.de/ forum/krankheiten-foren/gehirn-nerven/schwindel-leere-im-kopf-probleme-beimfokussieren, 2.1.2019) 
Beide Zitate machen sehr eindrücklich klar, dass die Ratsuchenden konstruktive ärztliche Empfehlung erwartet hätten, die jeweiligen Ärzte dazu aber (zumindest aus der Sicht der jeweiligen Patienten) nicht in der Lage waren. Die grundsätzliche Akzeptanz ärztlicher Empfehlungen wird aber gerade nicht hinterfragt, wenn man sich nach entsprechenden Diagnosen von Seiten der Ärzte sehnt; damit ist auch eindeutig, dass medizinisches Wissen trotz der negativen Erfahrung nicht in Frage gestellt wird.

Gelegentlich werden nach negativen Erfahrungen lediglich Experten ausgetauscht, was darauf hindeutet, dass kein grundsätzlicher Zweifel an medizinischem Wissen existiert:

7. Danach kam ein Spiessrutenlauf von Arzt zu Arzt. Alles wurde getestet. [...] Von den Ärzten fühlte ich mich schon lange nicht mehr Ernst genommen. Zudem sind drei meiner besten Freunde Ärzte, auch sie konnten mir nicht weiterhelfen. Die Verzweiflung wuchs. (https://www.gesundheit.de/forum/krankheiten-foren/magen-darm/wie-ich-meinenreizdarm-nach-3-jahren-dank-fmt-fecal-microbiota, 14.9.2018)

Beispiel 8 zeigt, dass in solchen Fällen dennoch die Bereitschaft existiert, notfalls auch einem unbekannten Arzt zu vertrauen. Offenbar richtet sich das Vertrauen auf seine demonstrierte Kompetenz. Medizinisches Wissen wird also gesucht; die Vertreter der Medizin sind jedoch offenbar nicht alle in der Lage, daraus sinnvolle Diagnosen abzuleiten.

8. In einem anderen Forum konnte ich zumindest von einem Arzt folgende, für mich logische, und der Sache noch am nähersten, Diagnose finden (https://www.gesundheit. $\mathrm{de} /$ forum/krankheiten-foren/hals-nasen-ohren/muskelzucken-im-ohr, 10.4.2018).

Dies bedeutet, dass sich der Autor ärztlicher Autorität nicht blind unterwirft; vielmehr muss die ärztliche Aussage für ihn „logisch[...]” sein.

Dass medizinisches Wissen und individuelles Verhalten der Ärzte oft sehr bewusst unterschieden werden, machen auch die folgenden Beispiele deutlich. Dabei wird immer wieder deutlich, dass klinische Studien grundsätzlich als sinnvolle Methode zur Generierung medizinischen Wissens akzeptiert sind.

Gegebenenfalls dienen sie dazu, ein Gegengewicht zum individuellen Fehlverhalten von Ärzten zu etablieren, auch in Beispiel 9: 
9. Da müsste man sich speziell gewisse Studien dazu genauer anschauen, ob [ein solches Vorgehen] wirklich sinnvoll ist. (https://www.gesundheit.de/forum/ernaehrungfitness-foren/diaet/fastentag, 12.4.2019)

Der Tipp eines/r Nutzers/in an den Fragesteller impliziert, dass,(klinische) Studien' das entscheidende Kriterium zur Vertrauenswürdigkeit darstellen. Auch bei Beispiel 10 ist klar, dass einer „Studie“ höchste Autorität zugebilligt wird - in jedem Fall deutlich höhere als „mein[em] Arzt“:

10. hi, mein Arzt hat mir gesagt, dass ich bei Sinusitis nicht inhalieren soll. Doch jetzt habe ich eine Studie gefunden, welche dieses ja im Grunde nahelegt. Oder ist eine Inhalation mit Nattokinase nicht mit dem Inkubatortest vergleichbar? [...] lieben Dank! (https://www.gesundheit.de/forum/krankheiten-foren/hals-nasen-ohren/inhalieren-beisinusitis, 12.1.2019)

Selbst der bereits recht frustrierend klingende Beitrag in Beispiel 11 impliziert keine Kritik an medizinischem Wissen:

11. ? Ich habe genau das gleiche Problem und kein Arzt findet die Lösung. (https:// www.gesundheit.de/forum/krankheiten-foren/schmerz/unerklaerlicher-knieschmerzaussen, 24.12.2018)

\subsubsection{Akzeptanz medizinischen Wissens und Suche nach Informationen unter Vermeidung der Ärzteschaft}

Nicht immer bleibt dies so. Relativ häufig finden sich auch Forumsbeiträge, die Kritik an Ärzten mit der Suche nach alternativen Ratschlägen, teilweise gar alternativen Autoritäten verbinden.

Auch hier gibt es Übergänge. Wenn bei Google gesucht wird, muss dies ebenfalls noch nicht eine Ablehnung medizinischen Wissens beziehungsweise ärztlichen Expertentums bedeuten.

12. Ich habe mich auf Google erkundigt da ich noch länger auf meinen Termin warten muss. (https://www.gesundheit.de/forum/krankheiten-foren/hals-nasen-ohren/tubenbel ueftungsstoerung, 9.12.2018) 
13. Ich habe natürlich auch gegoogelt, es würde die innere Analfistel vielleicht passen, aber ich habe keine Schmerzen... Sind es vielleicht doch Hämorrhoiden? (https://www. gesundheit.de/forum/krankheiten-foren/magen-darm/durchfallaehnliches-sekret-undblaehungen, 16.3.2019)

In den folgenden Beispielen sucht der/die Fragende Laienerfahrungen und -ratschläge als Reaktion auf offenbar unbefriedigende Aussagen des ärztlichen Experten:

14. Der HNO Arzt meinte viel Feuchtigkeit, Schmerzmittel und inhalieren und Stimme schonen, Also eigentlich nichts was ich nicht schon weiß. WENN JEMAND ERFAHRUNG HAT BITTE MELDET EUCH, ich bin wirklich frustriert, ich kann nicht reden und keinen Sport machen!! Wie krieg ich das weg???? LG und DANKE für Antworten (https://www. gesundheit.de/forum/krankheiten-foren/hals-nasen-ohren/kehlkopfentzuendungbrauche-hilfe, 12.1.2019)

15. ich habe seit fünf Jahren starke Verdauungsbeschwerden und weiß einfach nicht weiter. Die Ärzte leider auch nicht, daher hoffe ich, dass vlt. hier jemand eine Idee hat. (https://www.gesundheit.de/forum/krankheiten-foren/magen-darm/seit-fuenf-jahrenungeklaerter-durchfall, 19.3.2019)

Auch hier ist (noch) nicht ersichtlich, dass der Beitrag eine grundsätzliche Kritik medizinischen Wissens und ärztlicher Autorität impliziert; dennoch führt die Abwendung von diesem Arzt zu einer Suche nach Informationen, die nun außerhalb des ärztlichen Umfelds erhofft werden.

\subsubsection{Suche nach Alternativen}

Dass diese Suche nach Alternativen möglicherweise auch dazu führen kann, die ärztliche Autorität und die klassische Medizin in Frage zu stellen, macht Beispiel 16 deutlich:

16. Inzwischen bin ich aber zum gluck wieder kerngesund und habe mich als ganzheitlicher Health Coach ausbilden lassen, weil ich möglichst vielen bei Verdauungsproblemen helfen möchte. Ich weiss ja selbst, wie schlimm und einschränkend die Probleme sind. (https://www.gesundheit.de/forum/krankheiten-foren/magen-darm/ durchfall-seid-mehr-als-10-jahren, 28.3.2019) 
Es wird auch nicht immer klar, ob die Hinweise auf ,ganzheitliche' beziehungsweise alternative Heilmethoden fundiert erfolgen, wie die folgenden Beispiel verdeutlichen. Insbesondere bei Beispiel 18 klingt ein deutliches kommerzielles Interesse an.

17. Liebe Sandra, das hört sich für mich nach einem Pilz an. Du könntest es vielleicht mal mit verdünntem Teebaum-/ Oreganoöl betupfen. Das ist beides gegen Pilz. Bei Oreganoöl reicht ein Tropfen auf 1 TL Trägeröl. [Teebaumöl (dm) ] kannst du auch pur auftragen. Ich putze mir sogar mit 2 Tropfen Teebaumöl auf der Zahnpasta die Zähne. (https://www.gesundheit.de/forum/krankheiten-foren/haut/undefinierbarerhautausschlag-im-mundbereich, 14.4.2019)

18. Liebe Jessica Ich bin zufällig beim Stöbern auf deinen Beitrag gestoßen. Wie geht es dir aktuell mit deinem Herzen? [Ich agiere für eine Firma in der Stammzellenforschung, welche eine natürliche patentierte körpereigene Formel entwickelt hat.] Gesundung ist hier für den Menschen wieder möglich. Auch wenn dir die Symptome nicht erklärbar sind. Gerne gebe ich dir mehr Infos darüber. Schreib mir einfach eine E-Mail und wir unterhalten uns ein bisschen darüber. [...] Freue mich von dir zu lesen. Hab eine gesunde Zeit. Sonnige Grüße (https://www.gesundheit.de/forum/krankheiten-foren/herz-kreislauf /herzrasen-trotz-normalen-puls, 26.2.2019)

Im Korpus fehlen Angebote, die völlig die klinische Medizin verlassen. Esoterische Beiträge gibt es in diesem Kontext nicht.

\section{Diskussion}

Die vorliegende qualitative Analyse der Beiträge in dem Gesundheitsforum diente dazu herauszufinden, welche Einschätzungen über die Ärzteschaft und allgemein die Schulmedizin dort dominieren. Dabei zeigte sich, dass grundsätzlich ein großes Vertrauen in die Schulmedizin, wie auch in die Ärzteschaft dominiert. Natürlich ist ein Forum, in dessen Kontext Probleme diskutiert werden, von der Grundtendenz her kritisch und nach Alternativen suchend. Die Kritik beziehungsweise die Suche nach Alternativen geht aber im vorliegenden Korpus nie so weit, dass die Schulmedizin in Frage gestellt wird. Zwar findet sich häufig Kritik an (individuellen) Ärzten, denen dann aber in der Regel ein individuelles Versagen und damit eben eine schlechte Kenntnis der Schulmedizin vorgeworfen wird. Mithin bestätigt diese Kritik sogar das Grundvertrauen in die Medizin. 
Es lag bei einem Forum, in dem sich Nutzer treffen, um sich über Krankheiten zu informieren und Arztbesuche vor- oder nachzubereiten, nahe, zu vermuten, dass dort auch nach Alternativen gesucht wird und dass Nutzer, die von ärztlichen Reaktionen enttäuscht sind, sich eventuell gar von der Schulmedizin abwenden. Vor diesem Hintergrund ist das grundsätzliche Vertrauen in die Medizin also durchaus überraschend. Möglicherweise hängt dies damit zusammen, dass der Name des Forums und seine Erscheinungsart eine gewisse Nähe zur Schulmedizin suggerieren, die diejenigen abschreckt, die alternative Heilmethoden suchen. Bereits der Namen des Forums und der damit verbundenen Seriosität könnten die dort zum Ausdruck kommende Art und Weise der Kommunikation und ihre inhaltliche Ausrichtung prägen. Von daher wäre zu überprüfen, ob sich in anderen Foren andere Positionen finden lassen.

\section{Begutachtung: Extern begutachtet.}

Interessenkonflikt: Es besteht kein Interessenkonflikt.

Finanzielle Förderung: Dieser Beitrag wurde von keiner Institution finanziell unterstützt.

Peer-review: Externally peer-reviewed.

Conflict of Interest: The author has no conflict of interest to declare.

Grant Support: The author declared that this study has received no financial support.

\section{Literaturverzeichnis}

Angermüller, J., Bunzmann, K. \& Nonhoff, M. (Hrsg.) (2001). Diskursanalyse: Theorien, Methoden, Anwendungen.

Hamburg: Argument-Verlag

Alexa (2019). Top Sites in Germany. URL: https://www.alexa.com/topsites/countries/DE.

Bertelsmann-Stiftung (Ed.) (2018). Das Internet: Auch Ihr Ratgeber für Gesundheitsfragen? Bevölkerungsumfrage zur Suche von Gesundheitsinformationen im Internet und zur Reaktion der Ärzte. Gütersloh 2018, Abgerufen am 17. Januar 2020. URL: https://www.bertelsmann-stiftung.de/fileadmin/files/BSt/Publikationen/ GrauePublikationen/VV_Studie_Das-Internet-auch-Ihr-Ratgeber_Befragung.pdf.

Bitkom (2011). Das Internet wird zum Gesundheitsratgeber. Abgerufen am 17. Januar 2020. URL: http://Www. bitkom.org/de/markt_statistik/64026_69111.aspx.

Bromme, R., Jucks, R. \& Runde, A. (2005). Barriers and biases in computer-mediated expert-laypersoncommunication. In R. Bromme, F. W. Hesse \& H. Spada (Eds.) (2005). Barriers and Biases in Computer-Mediated Knowledge Communication. And How They May Be Overcome (S. 89-118). Dordrecht: Kluwer.

Busch, A. (2015). Medizindiskurse: Mediale Räume der Experten-Laien-Kommunikation. In A. Busch \& T. SpranzFogasy (Eds.). Handbuch Sprache in der Medizin. (S. 369-388). Berlin, New York: de Gruyter.

Dilthey, W. (1900/1964). Die Entstehung der Hermeneutik. In Dilthey, W (1964), Gesammelte Schriften, Band V. (S. 317-331) (Original: 1900). Göttingen: Vandenhoek \& Ruprecht. 
Ehrhardt, C. (2011). Höflichkeitsbegriffe - am Beispiel von Höflichkeit und Höflichkeitsbewusstsein in Internetforen. In C. Ehrhardt, E. Neuland \& H. Yamashita (Hgg.), Sprachliche Höflichkeit zwischen Etikette und kommunikativer Kompetenz (S. 27-44). Frankfurt/Main: Lang.

Ehrhardt, C. (2014). Politeness and face work in German forum communication. In K. Bedijs, G. Held, \& C. Maaß (Hrsg.), Face Work and Social Media. Münster: LIT.

Gadamer, H.-G. (1960). Wahrheit und Methode. Grundzüge einer philosophischen Hermeneutik. Tübingen: Mohr Siebeck Verlag.

Gillies, J. \& Cailliau, R. (2000). How the Web Was Born: The Story of the World Wide Web. Oxford, New York: Oxford University Press.

Kaltenborn, K.-F. (2001). Medizin- und gesundheitlicher Wissenstransfer durch Medien. In K. Hurrelmann \& A. Leppin (Eds.). Moderne Gesundheitskommunikation. Vom Aufklärungsgespräch zur E-Health. (S. 36-69). Bern: Huber.

Kassner, K. (2003). Soziale Deutungsmuster - über aktuelle Ansätze zur Erforschung kollektiver Sinnzusammenhänge. In S. Geideck \& W.-A. Liebert (Eds.). Sinnformeln. Linguistische und soziologische Analysen von Leitbildern, Metaphern und anderen kollektiven Orientierungsmustern. (pp. 37-57). Berlin. New York: de Gruyter.

Kleinke, S. (2015). Internetforen: Laiendiskurs Gesundheit. In A. Busch \& T. Spranz-Fogasy (Eds.). Handbuch Sprache in der Medizin. (S. 405-422). Berlin, New York: de Gruyter.

Kristiansen, S. \& Bonfadelli, H. (2013). E-Health: Gesundheit im Internet. In C. Rossmann \& M. Hastall (Eds.). Medien und Gesundheitskommunikation. Befunde, Entwicklungen, Herausforderungen. (S. 237-255). Baden-Baden: Nomos.

Kuckartz, Udo (2016). Qualitative Inhaltsanalyse. Methoden, Praxis, Computerunterstützung. 3. Auflage. Weinheim, Basel: Beltz Juventa.

Lippmann, W. (1922). Public Opinion. New York: Harcourt, Brace \& Co.

Myers, G. (2015). Social Media and professional Practice in Medical Twitter. In M. Gotti, S. Maci \& M. Sala (Eds.). Insights into Medical Communication. (S. 51-69). Bern: Lang.

O'Reilly, T. (September 30., 2005). What Is Web 2.0. Abgerufen am 17. Januar 2020. URL: https://Www.oreilly.com/ pub/a/web2/archive/what-is-web-20.html.

Rentel, N (2015). Sprachnormen, Community Building und Identity Management in der deutschen und französischen Forenkommunikation. Positiv wertende Kommentare in Diskussionsforen zu Schwangerschaft und Geburt. Bulletin VALS-ASLA, tome 2, 197-214.

Statista (2017). Volumen des ehealth-Marktes. Hamburg: Statistica. Abgerufen am 17. Januar 2020. URL: statistic id712698_volumen-des-ehealth-merktes-weltweit-bis-2020-pdf.

Statista (2019). Mediennutzung in Europa. Hamburg: Statistica. Abgerufen am 17. Januar 2020. URL: https://de. statista.com/statistik/studie/id/24564/dokument/mediennutzung-in-europa-statista-dossier.

www.gesundheit.de (2019). Abgerufen am 17. Januar 2020. Frankfurt: Alliance Healthcare Deutschland. 
